\title{
Multivariate Interpolation Functions of Higher-Order $q$-Euler Numbers and Their Applications
}

\author{
Hacer Ozden, ${ }^{1}$ Ismail Naci Cangul, ${ }^{1}$ and Yilmaz Simsek ${ }^{2}$ \\ ${ }^{1}$ Department of Mathematics, Faculty of Arts and Science, University of Uludag, Bursa 16059, Turkey \\ ${ }^{2}$ Department of Mathematics, Faculty of Arts and Science, University of Akdeniz, Antalya 07058, Turkey
}

Correspondence should be addressed to Hacer Ozden, hozden@uludag.edu.tr

Received 7 December 2007; Accepted 22 January 2008

Recommended by Paul Eloe

The aim of this paper, firstly, is to construct generating functions of $q$-Euler numbers and polynomials of higher order by applying the fermionic $p$-adic $q$-Volkenborn integral, secondly, to define multivariate $q$-Euler zeta function (Barnes-type Hurwitz $q$-Euler zeta function) and $l$-function which interpolate these numbers and polynomials at negative integers, respectively. We give relation between Barnes-type Hurwitz $q$-Euler zeta function and multivariate $q$-Euler $l$-function. Moreover, complete sums of products of these numbers and polynomials are found. We give some applications related to these numbers and functions as well.

Copyright ( $\odot 2008$ Hacer Ozden et al. This is an open access article distributed under the Creative Commons Attribution License, which permits unrestricted use, distribution, and reproduction in any medium, provided the original work is properly cited.

\section{Introduction, definitions, and notations}

Let $p$ be a fixed odd prime. Throughout this paper, $\mathbb{Z}_{p}, \mathbb{Q}_{p}, \mathbb{C}$, and $\mathbb{C}_{p}$ will, respectively, denote the ring of $p$-adic rational integers, the field of $p$-adic rational numbers, the complex number field, and the completion of the algebraic closure of $\mathbb{Q}_{p} . \mathbb{Z}_{+}=\mathbb{Z}^{+} \cup\{0\}$. Let $v_{p}$ be the normalized exponential valuation of $\mathbb{C}_{p}$ with $|p|_{p}=p^{-v_{p}(p)}=1 / p$ (cf. [1-28]). When we talk about $q$-extensions, $q$ is variously considered as an indeterminate, either a complex $q \in \mathbb{C}$, or a $p$-adic number $q \in \mathbb{C}_{p}$. If $q \in \mathbb{C}$, we assume that $|q|<1$. If $q \in \mathbb{C}_{p}$, then we assume $|q-1|_{p}<p^{-1 /(p-1)}$ so that $q^{x}=\exp (x \log q)$ for $|x|_{p} \leq 1$.

For a fixed positive integer $d$ with $(p, d)=1$, set

$$
\begin{gathered}
\mathbb{X}_{d}=\lim _{\overleftarrow{N}} \mathbb{Z} / d p^{N} \mathbb{Z}, \\
\mathbb{X}_{1}=\mathbb{Z}_{p},
\end{gathered}
$$




$$
\begin{gathered}
c \mathbb{X}^{*}=\bigcup_{\substack{0<a<d p \\
(a, p)=1}}\left(a+d p \mathbb{Z}_{p}\right), \\
a+d p^{N} \mathbb{Z}_{p}=\left\{x \in \mathbb{X}: x \equiv a\left(\bmod d p^{N}\right)\right\},
\end{gathered}
$$

where $a \in \mathbb{Z}$ satisfies the condition $0 \leq a<d p^{N}$ (cf. [1-28]).

The distribution $\mu_{q}\left(a+d p^{N} \mathbb{Z}_{p}\right)$ is given as

$$
\mu_{q}\left(a+d p^{N} \mathbb{Z}_{p}\right)=\frac{q^{a}}{\left[d p^{N}\right]_{q}}
$$

(cf. $[4,10])$.

We say that $f$ is a uniformly differentiable function at a point $a \in \mathbb{Z}_{p}$; we write $f \in$ $\mathrm{UD}\left(\mathbb{Z}_{p}\right)$ if the difference quotient

$$
F_{f}(x, y)=\frac{f(x)-f(y)}{x-y}
$$

has a limit $f^{\prime}(a)$ as $(x, y) \rightarrow(a, a)$. Let $f \in \operatorname{UD}\left(\mathbb{Z}_{p}\right)$. An invariant $p$-adic $q$-integral is defined by

$$
I_{q}(f)=\int_{\mathbb{Z}_{p}} f(x) d \mu_{q}(x)=\lim _{N \rightarrow \infty} \frac{1}{\left[p^{N}\right]_{q}} \sum_{x=0}^{p^{N}-1} f(x) q^{x}
$$

(cf. $[4,5,10,29,30])$.

The $q$-extension of $n \in \mathbb{N}$ is defined by

$$
[n]_{q}=\frac{1-q^{n}}{1-q}
$$

We note that $\lim _{q \rightarrow 1}[n]_{q}=n$.

Classical Euler numbers are defined by means of the following generating function:

$$
\frac{2}{e^{t}+1}=\sum_{n=0}^{\infty} E_{n} \frac{t^{n}}{n !}
$$

(cf. $[1-3,5,8,9,15,16,18-20,23,28,30]$ ), where $E_{n}$ denotes classical Euler numbers. These numbers are interpolated by the Euler zeta function which is defined as follows:

$$
\zeta_{E}(s)=\sum_{n=1}^{\infty} \frac{(-1)^{n}}{n^{s}}, \quad s \in \mathbb{C},
$$

(cf. $[8,9,24,25,28])$.

$q$-Euler numbers and polynomials have been studied by many mathematicians. These numbers and polynomials are very important in number theory, mathematical analysis and statistics, and the other areas. 
In [16], Ozden and Simsek constructed extensions of $q$-Euler numbers and polynomials. In [8], Kim et al. constructed new $q$-Euler numbers and polynomials which are different from Ozden and Simsek [16].

In [31], Kim gave a detailed proof of fermionic $p$-adic $q$-measures on $\mathbb{Z}_{p}$. He treated some interesting formulae related $q$-extension of Euler numbers and polynomials. He defined fermionic $p$-adic $q$-measures on $\mathbb{Z}_{p}$ as follows:

$$
\mu_{-q}\left(a+d p^{N} \mathbb{Z}_{p}\right)=\frac{(-q)^{a}}{\left[d p^{N}\right]_{-q}}
$$

where

$$
[n]_{-q}=\frac{1-(-q)^{n}}{1+q}
$$

(cf. $[1,31])$.

By using the fermionic $p$-adic $q$-measures, he defined the fermionic $p$-adic $q$-integral on $\mathbb{Z}_{p}$ as follows:

$$
I_{-q}(f)=\int_{\mathbb{Z}_{p}} f(x) d \mu_{-q}(x)=\lim _{N \rightarrow \infty} \frac{1}{\left[p^{N}\right]_{-q}} \sum_{x=0}^{p^{N}-1} f(x)(-q)^{x}
$$

(cf. [31]).

Observe that $I_{-q}(f)$ can be written symbolically as

$$
\lim _{q \rightarrow-q} I_{q}(f)=I_{-q}(f)
$$

(cf. [31]).

By using fermionic $p$-adic $q$-integral on $\mathbb{Z}_{p}$, Kim et al. [8] defined the generating function of the $q$-Euler numbers as follows:

$$
F_{q}(t)=\frac{q+1}{q e^{t}+1}=\sum_{n=0}^{\infty} E_{n, q} \frac{t^{n}}{n !}
$$

where $E_{n, q}$ denotes $q$-Euler numbers.

Witt's formula of $E_{n}(x, q)$ was given by Kim et al. [8]:

$$
E_{n}(x, q)=\int_{\mathbb{Z}_{p}}(x+y)^{n} d \mu_{-q}(y)
$$

where $q \in \mathbb{C}_{p}$ and $|1-q|_{p}<1$.

In [16], Ozden and Simsek defined generating function of $q$-Euler numbers by

$$
F(t, q)=\frac{2}{q e^{t}+1}=\frac{2}{q+1} F_{q}(t)
$$


In $[7,9]$, Kim defined $q$ - $l$-functions and $q$-multiple $l$-functions. He also gave many applications of these functions.

We summarize our paper as follows. In Section 2, we give some fundamental properties of the $q$-Euler numbers and polynomials. We also give some relations related to these numbers and polynomials. By using generating functions of $q$-Euler numbers and polynomials of higher order, we define multivariate $q$-Euler zeta function (Barnes-type Hurwitz $q$-Euler zeta function) and $l$-function which interpolate these numbers and polynomials at negative integers. We also give contour integral representation of these functions. In Section 3, we find relation between $l_{E, q}^{(r)}(s, x)$ and $\zeta_{q, E}^{(r)}(s, x)$. By using these relations, we obtain distribution relations of the generalized $q$-Euler numbers and polynomials of higher order. In Section 4, we find complete sums of products of these numbers and polynomials. We also give some applications related to these numbers and functions.

\section{Some properties of $q$-Euler numbers and polynomials}

For $q \in \mathbb{C}$ with $|q|<1$,

$$
F_{q}(t)=\frac{q+1}{q e^{t}+1}=\sum_{n=0}^{\infty} E_{n, q} \frac{t^{n}}{n !}
$$

(cf. [8]), where $E_{n, q}$ denotes the $q$-Euler number and $|t+\log q|<\pi$.

Observe that by (2.1) we have

$$
\frac{q+1}{q e^{t}+1}=\frac{1+q^{-1}}{e^{t}+q^{-1}}=\sum_{n=0}^{\infty} H_{n}\left(-q^{-1}\right) \frac{t^{n}}{n !} .
$$

From (2.1) and (2.2), we note that $H_{n}\left(-q^{-1}\right)=E_{n, q}$, where $H_{n}\left(-q^{-1}\right)$ are called Frobenius Euler numbers (cf. $[27,28]$ ). [8]:

The $q$-Euler polynomials are also defined by means of the following generating function

$$
F_{q}(t, x)=\frac{q+1}{q e^{t}+1} e^{x t}=\sum_{n=0}^{\infty} E_{n, q}(x) \frac{t^{n}}{n !}
$$

where $|t+\log q|<\pi$;

$$
\begin{aligned}
\frac{q+1}{q e^{t}+1} e^{x t} & =e^{E_{q}(x) t}, \\
(q+1) e^{x t} & =q e^{\left(1+E_{q}(x)\right) t}+e^{E_{q}(x) t}, \\
(q+1) \sum_{n=0}^{\infty} x^{n} \frac{t^{n}}{n !} & =\sum_{n=0}^{\infty}\left(q\left(1+E_{q}(x)\right)^{n}+E_{n, q}(x)\right) \frac{t^{n}}{n !} .
\end{aligned}
$$

By comparing the coefficients of $t^{n}$ on both sides of the above equation, we have the following theorem. 
Hacer Ozden et al.

Theorem 2.1. Let $n$ be nonnegative integer. Then

$$
q\left(1+E_{q}(x)\right)^{n}+E_{n, q}(x)=(q+1) x^{n}
$$

with the usual convention about replacing $E_{q}^{n}(x)$ by $E_{n, q}(x)$.

By using (2.5), we have

$$
E_{n, q}(x)+q \sum_{k=0}^{n}\left(\begin{array}{l}
n \\
k
\end{array}\right) E_{k, q}(x)=(q+1) x^{n}
$$

From (2.3), by applying Cauchy product and using (2.1), we also obtain

$$
\left(\sum_{n=0}^{\infty} E_{n, q} \frac{t^{n}}{n !}\right)\left(\sum_{n=0}^{\infty} x^{n} \frac{t^{n}}{n !}\right)=\sum_{n=0}^{\infty}\left(\sum_{k=0}^{\infty}\left(\begin{array}{l}
n \\
k
\end{array}\right) x^{n-k} E_{k, q}\right) \frac{t^{n}}{n !}=\sum_{n=0}^{\infty} E_{n, q}(x) \frac{t^{n}}{n !} .
$$

By comparing the coefficients of $t^{n}$ on both sides of the above equation, we have

$$
E_{n, q}(x)=\sum_{k=0}^{n}\left(\begin{array}{l}
n \\
k
\end{array}\right) x^{n-k} E_{k, q}
$$

(cf. $[8,14]$ ).

By using Theorem 2.1 and [8, equation (3)], we obtain

$$
\begin{aligned}
E_{n}(x, q) & =\int_{\mathbb{Z}_{p}}(x+y)^{n} d \mu_{-q}(y) \\
& =\lim _{N \rightarrow \infty} \frac{1}{\left[j p^{N}\right]_{-q}} \sum_{x=0}^{j p^{N}-1}(x+y)^{n}(-q)^{x} \\
& =\frac{1}{[j]_{-q}} \lim _{N \rightarrow \infty} \frac{1}{\left[p^{N}\right]_{(-q)^{j}}} \sum_{x=0}^{j-1} \sum_{x=0}^{j p^{N}-1}(a+j x+y)^{n}(-q)^{a+j x} \\
& =\frac{j^{n}}{[j]_{-q}} \lim _{N \rightarrow \infty} \frac{1}{\left[p^{N}\right]_{(-q)^{j}}} \sum_{a=0}^{j-1}(-q)^{a^{N}} \sum_{x=0}^{p^{N}-1}\left(\frac{a+y}{j}+x\right)^{n}\left((-q)^{j}\right)^{x} \\
& =\frac{j^{n}}{[j]_{-q}} \sum_{a=0}^{j-1}(-q)^{a} \lim _{N \rightarrow \infty} \frac{1}{\left[p^{N}\right]_{(-q)^{j}}} \sum_{x=0}^{p^{N}-1}\left(\frac{a+y}{j}+x\right)^{n}\left((-q)^{j}\right)^{x} \\
& =\frac{j^{n}}{[j]_{-q}} \sum_{a=0}^{j-1}(-q)^{a} E_{n}\left(\frac{a+y}{j}, q^{j}\right) .
\end{aligned}
$$


By using the above equation, we arrive at the following theorem.

Theorem 2.2. Let $j$ be odd. Then

$$
E_{n, q}(x)=\frac{(q+1) j^{n}}{q^{j}+1} \sum_{a=0}^{j-1}(-1)^{a} q^{a} E_{n, q^{j}}\left(\frac{a+x}{j}\right) .
$$

By simple calculation in (2.3), Ryoo et al. [14] give another proof of Theorem 2.2,which is given as follows: let $j$ be odd;

$$
\begin{aligned}
\sum_{n=0}^{\infty} E_{n}(x) \frac{t^{n}}{n !} & =\frac{q+1}{q e^{t}+1} e^{x t} \\
& =\frac{q+1}{1+q^{j} e^{j t}} \sum_{a=0}^{j-1}(-1)^{a} q^{a} e^{a t} e^{x t} \\
& =(q+1) \sum_{a=0}^{j-1}(-1)^{a} q^{a}\left(\frac{e^{(a+x) t}}{1+q^{j} e^{j t}}\right) \frac{q^{j}+1}{q^{j}+1} \\
& =\sum_{n=0}^{\infty}\left(\frac{q+1}{q^{j}+1} j^{n} \sum_{a=0}^{d-1}(-1)^{a} q^{a} E_{n, q^{j}}\left(\frac{a+x}{j}\right)\right) \frac{t^{n}}{n !}
\end{aligned}
$$

By comparing the coefficients of $t^{n}$ on both sides of the above equation, we have Theorem 2.2.

By substituting $x=n$, with $n \in \mathbb{Z}_{+}$into (2.3), then we have

$$
F_{q}(t, n)=\frac{q+1}{q e^{t}+1} e^{n t}=\sum_{k=0}^{\infty} E_{k, q}(n) \frac{t^{k}}{k !}
$$

Thus,

$$
\begin{aligned}
F_{q}(t) & -q^{n}(-1)^{n} F_{q}(t, n) \\
& =(q+1) \sum_{l=0}^{\infty}(-1)^{l} q^{l} e^{l t}-(q+1) \sum_{l=0}^{\infty}(-1)^{l+n} q^{l+n} e^{t(l+n)} \\
& =(q+1) \sum_{l=0}^{n-1}(-1)^{l} q^{l} e^{l t}+(q+1) \sum_{l=0}^{\infty}(-1)^{l+n} q^{l+n} e^{t(l+n)}-(q+1) \sum_{l=0}^{\infty}(-1)^{l+n} q^{l+n} e^{t(l+n)} .
\end{aligned}
$$

Hence, by (2.13), we have

$$
F_{q}(t)-q^{n}(-1)^{n} F_{q}(t, n)=(q+1) \sum_{l=0}^{n-1}(-1)^{l} q^{l} e^{l t}
$$

By the generating function of $q$-Euler numbers and polynomials and by (2.14), we see that

$$
\sum_{m=0}^{\infty}\left(E_{m, q}-q^{n}(-1)^{n} E_{m, q}(n)\right) \frac{t^{m}}{m !}=\sum_{m=0}^{\infty}\left((q+1) \sum_{l=0}^{n-1} q^{l}(-1)^{l} l^{m}\right) \frac{t^{m}}{m !} .
$$


By comparing the coefficients of $t^{n}$ on both sides of (2.15), we obtain the following alternating sums of powers of consecutive $q$-integers as follows.

Theorem 2.3 (see [14]). Let $n \in \mathbb{Z}_{+}$. Then

$$
\frac{E_{m, q}-q^{n}(-1)^{n} E_{m, q}(n)}{q+1}=\sum_{l=0}^{n-1} q^{l}(-1)^{l} l^{m} .
$$

Remark 2.4. Proof of Theorem 2.3 is similar to that of [14]. If we take $q \rightarrow 1$ in (2.16), we have

$$
\frac{E_{m}-(-1)^{n} E_{m}(n)}{2}=\sum_{l=0}^{n-1}(-1)^{l} l^{m}
$$

The above formula is well known in the number theory and its applications.

Remark 2.5. Generating function of the $q$-Euler numbers in this paper is different than that in $[29,31]$. It is same as in [8]. Consequently, all these generating functions in $[8,16,29,31]$ produce different-type $q$-Euler numbers. But we observe that all these generating functions were obtained by the same fermionic $p$-adic $q$-measures on $\mathbb{Z}_{p}$ and the fermionic $p$-adic $q$ integral on $\mathbb{Z}_{p}$; for applications of this integral and measure see for detail $[2,4,8,14-19,23,25$, 29-31].

Now, we consider $q$-Euler numbers and polynomials of higher order as follows:

$$
\underbrace{\left(\frac{q+1}{q e^{t}+1}\right)\left(\frac{q+1}{q e^{t}+1}\right) \cdots\left(\frac{q+1}{q e^{t}+1}\right)}_{r \text { times }}=\left(\frac{q+1}{q e^{t}+1}\right)^{r}=\sum_{n=0}^{\infty} E_{n, q}^{(r)} \frac{t^{n}}{n !},
$$

where $E_{n, q}^{(r)}$ are called $q$-Euler numbers of order $r$. We also consider $q$-Euler polynomials of order $r$ as follows:

$$
\left(\frac{q+1}{q e^{t}+1}\right)\left(\frac{q+1}{q e^{t}+1}\right) \cdots\left(\frac{q+1}{q e^{t}+1}\right) e^{t x}=\frac{(q+1)^{r} e^{t x}}{\left(q e^{t}+1\right)^{r}}=\sum_{n=0}^{\infty} E_{n, q}^{(r)}(x) \frac{t^{n}}{n !},
$$

where $|t+\log q|<\pi$. From these generating functions of $q$-Euler numbers and polynomials of higher order, we construct multiple $q$-Euler zeta functions. First, we investigate the properties of generating function of $q$-Euler polynomials of higher order as follows:

$$
\begin{aligned}
F_{q}^{(r)}(t, x) & =\underbrace{\frac{q+1}{q e^{t}+1} \frac{q+1}{q e^{t}+1} \cdots \frac{q+1}{q e^{t}+1} e^{t x}}_{r \text { times }} \\
& =\sum_{j=0}^{r}\left(\begin{array}{c}
r \\
j
\end{array}\right) q^{j} e^{t x} \sum_{n_{1}=0}^{\infty}(-1)^{n_{1}} q^{n_{1}} e^{n_{1} t} \ldots \sum_{n_{r}=0}^{\infty}(-1)^{n_{r}} q^{n_{r}} e^{n_{r} t} \\
& =\sum_{n_{1}, n_{2}, \ldots, n_{r}=0}^{\infty} \sum_{j=0}^{r}\left(\begin{array}{c}
r \\
j
\end{array}\right)(-1)^{n_{1}+\cdots+n_{r}} q^{j+n_{1}+\cdots+n_{r}} e^{\left(n_{1}+\cdots+n_{r}+x\right) t} \\
& =\sum_{n=0}^{\infty} E_{n, q}^{(r)}(x) \frac{t^{n}}{n !} .
\end{aligned}
$$


By applying Mellin transformation to (2.20), we have

$$
\frac{1}{\Gamma(s)} \int_{0}^{\infty} t^{s-1} F_{q}^{(r)}(t, x) d t=\sum_{n_{1}, n_{2}, \ldots, n_{r}=0}^{\infty} \sum_{j=0}^{r}\left(\begin{array}{l}
r \\
j
\end{array}\right) \frac{1}{\Gamma(s)} \int_{0}^{\infty} t^{s}(-1)^{n_{1}+\cdots+n_{r}} q^{j+n_{1}+\cdots+n_{r}} e^{\left(n_{1}+\cdots+n_{r}+x\right) t} d t .
$$

After some elementary calculations, we obtain

$$
\begin{aligned}
\frac{1}{\Gamma(s)} \int_{0}^{\infty} t^{s-1} F_{q}^{(r)}(-t, x) d t & =\sum_{j=0}^{r}\left(\begin{array}{c}
r \\
j
\end{array}\right) q^{j} \sum_{n_{1}, n_{2}, \ldots, n_{r}=0}^{\infty} \sum_{n_{1}, n_{2}, \ldots, n_{r}=0}^{\infty} \frac{(-1)^{n_{1}+\cdots+n_{r}} q^{n_{1}+\cdots+n_{r}}}{\left(n_{1}+\cdots+n_{r}+x\right)^{s}} \\
& =\sum_{n_{1}, n_{2}, \cdots, n_{r}=0}^{\infty} \sum_{j=0}^{r}\left(\begin{array}{c}
r \\
j
\end{array}\right) \frac{(-1)^{n_{1}+\cdots+n_{r}} q^{j+n_{1}+\cdots+n_{r}}}{\left(n_{1}+\cdots+n_{r}+x\right)^{s}} .
\end{aligned}
$$

From (2.22), we define the analytic function which interpolates higher-order $q$-Euler numbers at negative integers as follows.

Definition 2.6. For $s \in \mathbb{C}, x \in \mathbb{R}(0<x \leq 1)$, one defines

$$
\zeta_{q, E}^{(r)}(s, x)=\sum_{n_{1}, n_{2}, \cdots, n_{r}=0}^{\infty} \sum_{j=0}^{r}\left(\begin{array}{l}
r \\
j
\end{array}\right) \frac{(-1)^{n_{1}+\cdots+n_{r}} q^{j+n_{1}+\cdots+n_{r}}}{\left(n_{1}+\cdots+n_{r}+x\right)^{s}} .
$$

$\zeta_{q, E}^{(r)}(s, x)$ is called Barnes-type Hurwitz $q$-Euler zeta function.

Remark 2.7. By applying the $k$ th derivative operator $d^{k} /\left.d t^{k}\right|_{t=0}$ on both sides of (2.20), we have

$$
E_{n, q}^{(r)}(x)=\left.\frac{d^{k}}{d t^{k}} F_{q}^{r}(t, x)\right|_{t=0}=\sum_{n_{1}, n_{2}, \ldots, n_{r}=0}^{\infty} \sum_{j=0}^{r}\left(\begin{array}{l}
r \\
j
\end{array}\right)(-1)^{n_{1}+\cdots+n_{r}} q^{j+n_{1}+\cdots+n_{r}}\left(n_{1}+\cdots+n_{r}+x\right)^{k} .
$$

By using the above equation, Ryoo et al. [14] and Simsek [23] also define (2.23).

By substituting $s=-k, k \in \mathbb{Z}_{+}$into (2.23) and using (2.24), after some calculations, we arrive at the following theorem.

Theorem 2.8. Let $k \in \mathbb{Z}_{+}$. Then

$$
\zeta_{q, E}^{(r)}(-k, x)=E_{n, q}^{(r)}(x)
$$

Observe that the function $\zeta_{q, E}^{(r)}(s, x)$ interpolates $E_{n, q}^{(r)}(x)$ polynomial at negative integers. By using the complex integral representation of generating function of the polynomials $E_{n, q}^{(r)}(x)$, we have

$$
\frac{1}{\Gamma(s)} \oint_{C} t^{s-1} F_{q}^{(r)}(-t, x) d t=\sum_{n=0}^{\infty} \frac{(-1)^{n} E_{n, q}^{(r)}(x)}{n !} \frac{1}{\Gamma(s)} \oint_{C} t^{n+s-1} d t,
$$

where $C$ is Hankel's contour along the cut joining the points $z=0$ and $z=\infty$ on the real axis, which starts from the point at $\infty$, encircles the origin $(z=0)$ once in the positive (counterclockwise) direction, and returns to the point at $\infty$ (see for detail [13, 17, 25, 28]). By using (2.26) and Cauchy-Residue theorem, then we arrive at (2.25). 
Remark 2.9. $\zeta_{q, E}^{(r)}(s, 1)=\zeta_{q, E}^{(r)}(s)$ is called Barnes-type $q$-Euler zeta function; see for detail [14]. $\zeta_{q, E}^{(r)}(s, x)$ is an analytic function in whole complex s-plane. For $s \in C$,

$$
\zeta_{E}^{r}(s, x)=\lim _{q \rightarrow 1} \zeta_{q, E}^{r}(s, x)=2^{r} \sum_{n_{1}, n_{2}, \ldots, n_{r}=0}^{\infty} \frac{(-1)^{n_{1}+\cdots+n_{r}}}{\left(n_{1}+\cdots+n_{r}+x\right)^{s}} .
$$

If $r=1$ in the above equation, we have

$$
\zeta_{E}(s, x)=2 \sum_{n=0}^{\infty} \frac{(-1)^{n}}{(n+x)^{s}} .
$$

The function $\zeta_{E}(s, x)$ is known as classical Hurwitz-type zeta function which interpolates classical Euler numbers at negative integers, cf. [28].

Let $x$ be Dirichlet's character with conductor $d \in \mathbb{Z}^{+}$. The generalized $q$-Euler numbers attached to $x$ of higher order are defined by

$$
F_{q, X}(t)=\frac{(q+1) \sum_{a=1}^{d}(-1)^{a} q^{a} X(a) e^{t a}}{q^{d} e^{d t}+1}=\sum_{n=0}^{\infty} E_{n, X} \frac{t^{n}}{n !}
$$

(cf. [8]), where $|t+\log d|<\pi$. The $q$-Euler numbers attached to $x$ of higher order are defined by

$$
\begin{aligned}
F_{q, X}^{(r)}(t) & =\left(\frac{(q+1) \sum_{a=1}^{d}(-1)^{a} q^{a} X(a) e^{t a}}{q^{d} e^{d t}+1}\right)^{r} \\
& =\sum_{n=0}^{\infty} E_{n, q, x}^{(r)} \frac{t^{n}}{n !} .
\end{aligned}
$$

From (2.30), we obtain

$$
\begin{aligned}
F_{q, X}^{(r)}(t) & =\sum_{n_{1}, n_{2}, \ldots, n_{r}=1}^{\infty} \sum_{j=0}^{r}\left(\begin{array}{l}
r \\
j
\end{array}\right)(-1)^{n_{1}+\cdots+n_{r}} q^{j+n_{1}+\cdots+n_{r}} e^{\left(n_{1}+\cdots+n_{r}\right) t} \prod_{k=1}^{r} X\left(n_{k}\right) \\
& =\sum_{n=0}^{\infty} E_{n, q, x}^{(r)} \frac{t^{n}}{n !} .
\end{aligned}
$$

By applying the $k$ th derivative operator $d^{k} /\left.d t^{k}\right|_{t=0}$ in (2.31), we have

$$
\begin{gathered}
E_{k, q, x}^{(r)}=\left.\frac{d^{k}}{d t^{k}} F_{q, x}^{(r)}(t)\right|_{t=0} \\
=\sum_{n_{1}, n_{2}, \ldots, n_{r}=1}^{\infty} \sum_{j=0}^{r}\left(\begin{array}{l}
r \\
j
\end{array}\right)(-1)^{n_{1}+\cdots+n_{r}} q^{j+n_{1}+\cdots+n_{r}}\left(n_{1}+\cdots+n_{r}\right)^{k} \prod_{k=1}^{r} X\left(n_{k}\right) .
\end{gathered}
$$

By using (2.32), we define Dirichlet-type multiple Euler $q$ - $l$-function as follows.

Definition 2.10. Let $s \in \mathbb{C}$;

$$
l_{E, q}^{(r)}(s, X)=\sum_{n_{1}, n_{2}, \cdots, n_{r}=1}^{\infty} \sum_{j=0}^{r}\left(\begin{array}{l}
r \\
j
\end{array}\right) \frac{(-1)^{n_{1}+\cdots+n_{r}} q^{j+n_{1}+\cdots+n_{r}} \prod_{k=1}^{r} \chi\left(n_{k}\right)}{\left(n_{1}+\cdots+n_{r}\right)^{s}} .
$$


Remark 2.11. $l_{E, q}^{(r)}(s, X)$ is an analytic function in the whole complex s-plane. From the above definition,

$$
l_{E}^{(r)}(s, \chi)=\lim _{q \rightarrow 1} l_{E, q}^{(r)}(s, \chi)=\sum_{n_{1}, n_{2}, \ldots, n_{r}=1}^{\infty} \frac{2(-1)^{n_{1}+\cdots+n_{r}} \prod_{k=1}^{r} \chi\left(n_{k}\right)}{\left(n_{1}+\cdots+n_{r}\right)^{s}} .
$$

For $r=1$ in the above equation, we have

$$
l_{E}(s, \chi)=\sum_{n=1}^{\infty} \frac{2(-1)^{n} \chi(n)}{n^{s}} .
$$

This function is called Euler $l$-function.

Here, we observe that by applying Mellin transformation to (2.31), we obtain

$$
\frac{1}{\Gamma(s)} \int_{0}^{\infty} t^{s-1} F_{q, X}^{(r)}(-t) d t=l_{E, q}^{(r)}(s, x) .
$$

This gives us another definition of (2.32). theorem.

By substituting $s=-k, k \in \mathbb{Z}_{+}$into (2.33) and using (2.32), we arrive at the following

Theorem 2.12. Let $k \in \mathbb{Z}_{+}$. Then

$$
l_{E, q}^{(r)}(-k, \chi)=E_{k, q, x}^{(r)} .
$$

We note that

$$
\lim _{q \rightarrow 1} l_{E, q}^{(r)}(-k, X)=l_{E}^{(r)}(-k, \chi)=E_{n, X}^{(r)}
$$

where $E_{n, x}$ are called classical Euler numbers attached to $x$ of higher order, cf. [28]. By using (2.26), (2.36), we obtain another proof of (2.37).

3. Relation between $l_{E, q}^{(r)}(s, \chi)$ and $\zeta_{q, E}^{(r)}(s, x)$

Substituting $n_{j}=a_{j}+m_{j} f$, where $m_{j}=0,1,2,3, \ldots, \infty$ and $a_{j}=1,2, \ldots, f$, where $\chi\left(a_{j}+m_{j} f\right)=$ $x\left(a_{j}\right)$ and $f$ is odd conductor of $x, 1 \leq j \leq r$, into (2.33), we have

$$
\begin{aligned}
l_{E, q}^{(r)}(s, X)= & (1+q)^{r} \sum_{a_{1}, a_{2}, \ldots, a_{r}=1}^{f} \sum_{m_{1}, m_{2}, \ldots, m_{r}=0}^{\infty} \frac{(-1)^{a_{1}+m_{1} f+\cdots+a_{r}+m_{r} f} q^{a_{1}+m_{1} f+\cdots+a_{r}+m_{r} f} \prod_{k=1}^{r} \chi\left(a_{k}+m_{k} f\right)}{\left(a_{1}+m_{1} f+\cdots+a_{r}+m_{r} f\right)^{s}} \\
= & \frac{(1+q)^{r} f^{-s}}{\left(1+q^{f}\right)^{r}} \sum_{a_{1}, a_{2}, \ldots, a_{r}=1}^{f}(-1)^{a_{1}+\cdots+a_{r}} q^{a_{1}+\cdots+a_{r}} \prod_{k=1}^{r} x\left(a_{k}\right) \\
& \times\left(1+q^{f}\right)^{r} \sum_{m_{1}, m_{2}, \ldots, m_{r}=0}^{\infty} \frac{(-1)^{m_{1}+\cdots+m_{r}} q^{f v+m_{1} f+\cdots+m_{r} f}}{\left(\frac{a_{1}+\cdots+a_{r}}{f}+m_{1}+\cdots+m_{r}\right)^{s}} .
\end{aligned}
$$

By substituting (2.23) into the above equation, we arrive at the following theorem. 
Theorem 3.1. Let $x$ be a Dirichlet character with conductor $f(=$ odd). Then

$$
l_{E, q}^{(r)}(s, X)=\frac{(1+q)^{r} f^{-s}}{\left(1+q^{f}\right)^{r}} \sum_{a_{1}, a_{2}, \ldots, a_{r}=1}^{f}(-1)^{a_{1}+\cdots+a_{r}} q^{a_{1}+\cdots+a_{r}} \prod_{k=1}^{r} \chi\left(a_{k}\right) \zeta_{q^{f}, E}^{(r)}\left(s, \frac{a_{1}+\cdots+a_{r}}{f}\right) .
$$

By substituting $s=-k, k \in \mathbb{Z}$, into (3.2), we obtain

$$
l_{E, q}^{(r)}(-k, \chi)=\frac{(1+q)^{r} f^{k}}{\left(1+q^{f}\right)^{r}} \sum_{a_{1}, a_{2}, \ldots, a_{r}=1}^{f}(-1)^{a_{1}+\cdots+a_{r}} q^{a_{1}+\cdots+a_{r}} \prod_{k=1}^{r} \chi\left(a_{k}\right) \zeta_{q^{f}, E}^{(r)}\left(-k, \frac{a_{1}+\cdots+a_{r}}{f}\right) .
$$

By using (2.25) and (2.37) in the above equation, we obtain distribution relation of the $q$-Euler numbers attached to $X$ of higher order, $E_{k, q, x^{\prime}}^{(r)}$ which is given as follows.

Theorem 3.2. The following holds:

$$
E_{k, q, X}^{(r)}=\frac{(1+q)^{r} f^{k}}{\left(1+q^{f}\right)^{r}} \sum_{a_{1}, a_{2}, \ldots, a_{r}=1}^{f}(-1)^{a_{1}+\cdots+a_{r}} q^{a_{1}+\cdots+a_{r}} \prod_{k=1}^{r} x\left(a_{k}\right) E_{n, q^{f}}^{(r)}\left(\frac{a_{1}+\cdots+a_{r}}{f}\right) .
$$

\section{Multivariate $p$-adic fermionic $q$-integral on $\mathbb{Z}_{p}$ associated with higher-order $q$-Euler numbers}

In [14], Ryoo et al. defined $q$-extension of Euler numbers and polynomials of higher order. They studied Barnes-type $q$-Euler zeta functions. They also derived sums of products of $q$-Euler numbers and polynomials by using fermionic $p$-adic $q$-integral. In this section, we assume that $q \in \mathbb{C}_{p}$ with $|1-q|_{p}<1$. By using (1.4), the $p$-adic fermionic $q$-integral on $\mathbb{Z}_{p}$ is defined by

$$
\begin{aligned}
I_{-q}(f) & =\int_{\mathbb{Z}_{p}} f(x) d \mu_{-q}(x) \\
& =\lim _{N \rightarrow \infty} \frac{1}{\left[p^{N}\right]_{-q}} \sum_{x=0}^{p^{N}-1} f(x)(-q)^{x} .
\end{aligned}
$$

From this integral equation, we have (see $[1,2,4]$ )

$$
q I_{-q}\left(f_{1}\right)+I_{-q}(f)=(q+1) f(0),
$$

where $f_{1}(x)=f(x+1)$. If we take $f(x)=e^{t x}$ in (4.2), we have

$$
I_{-q}\left(e^{t x}\right)=\int_{\mathbb{Z}_{p}} e^{t x} d \mu_{-q}(x)=\frac{q+1}{q e^{t}+1}=\sum_{n=0}^{\infty} E_{n, q} \frac{t^{n}}{n !}
$$

(cf. [8]). 
Now we are ready to give multivariate $p$-adic fermionic $q$-integral on $\mathbb{Z}_{p}$ as follows (see for detail [14]). Let

$$
\begin{gathered}
\int_{\mathbb{Z}_{p}^{r}}=\underbrace{\int_{\mathbb{Z}_{p}} \int_{\mathbb{Z}_{p}} \cdots \int_{\mathbb{Z}_{p}}}_{r \text { times }} \\
\int_{\mathbb{Z}_{p}^{r}} e^{t\left(x_{1}+\cdots+x_{r}\right)} d \mu_{-q}\left(x_{1}\right) \cdots d \mu_{-q}\left(x_{r}\right)=\underbrace{\left(\frac{q+1}{q e^{t}+1}\right) \cdots\left(\frac{q+1}{q e^{t}+1}\right)}_{r \text { times }}=\sum_{n=0}^{\infty} E_{n, q}^{(r)} \frac{t^{n}}{n !} .
\end{gathered}
$$

From (4.4), we obtain Witt's formula for $q$-Euler numbers of higher order as follows.

Theorem 4.1 (see $[14]$ ). Let $k \in \mathbb{Z}_{+}$. Then

$$
\int_{\mathbb{Z}_{p}^{r}}\left(x_{1}+\cdots+x_{r}\right)^{n} d \mu_{-q}\left(x_{1}\right) \cdots d \mu_{-q}\left(x_{r}\right)=E_{n, q}^{(r)}
$$

By (4.4), we obtain

$$
\int_{\mathbb{Z}_{p}^{r}} e^{t\left(x_{1}+\cdots+x_{r}+x\right)} d \mu_{-q}\left(x_{1}\right) \cdots d \mu_{-q}\left(x_{r}\right)=\underbrace{\frac{e^{x t}(q+1)^{r}}{\left(q e^{t}+1\right) \cdots\left(q e^{t}+1\right)}}_{r \text { times }}=\sum_{n=0}^{\infty} E_{n, q}^{(r)}(x) \frac{t^{n}}{n !} .
$$

Theorem 4.2 (multinomial theorem). The following holds:

$$
\left(\sum_{j=1}^{v} x_{j}\right)^{n}=\sum_{\substack{l_{1}, l_{2}, \ldots, l_{v} \geq 0 \\
l_{1}+l_{2}+\cdots+l_{v}=n}}\left(\begin{array}{c}
n \\
l_{1}, l_{2}, \ldots, l_{v}
\end{array}\right) \prod_{a=1}^{v} x_{a}^{l_{a}}
$$

where $\left(\begin{array}{c}n \\ l_{1}, l_{2}, \ldots, l_{v}\end{array}\right)$ are the multinomial coefficients, which are defined by

$$
\left(\begin{array}{c}
n \\
l_{1}, l_{2}, \ldots, l_{v}
\end{array}\right)=\frac{n !}{l_{1} ! l_{2} ! \ldots l_{v} !}
$$

(cf. $[32,33])$.

Now we give a main theorem of this section, which is called complete sums of products of $q$-Euler polynomials of higher order.

Theorem 4.3. For positive integers $n, r$, one has

$$
E_{n, q}^{(r)}\left(y_{1}+y_{2}+\cdots+y_{r}\right)=\sum_{\substack{l_{1}, l_{2}, \ldots, l_{r} \geq 0 \\
l_{1}+l_{2}+\cdots, l_{r}=n}}\left(\begin{array}{c}
n \\
l_{1}, l_{2}, \ldots, l_{r}
\end{array}\right) \prod_{j=1}^{r} E_{l_{j}, q}\left(y_{j}\right),
$$

where $\left(\begin{array}{c}n \\ l_{1}, l_{2}, \ldots, l r\end{array}\right)$ is the multinomial coefficient. 
Proof. The proof of this theorem is similar to that of [23]. By using Taylor series of $e^{t x}$ into (4.6), and $x$ by $y_{1}+y_{2}+\cdots+y_{r}$, then we have

$$
E_{n, q}^{(r)}\left(y_{1}+y_{2}+\cdots+y_{r}\right)=\int_{\mathbb{Z}_{p}} \cdots \int_{\mathbb{Z}_{p}}\left(\sum_{j=1}^{r}\left(y_{j}+x_{j}\right)\right)^{n} \prod_{j=1}^{r} d \mu_{-q}\left(x_{j}\right) .
$$

By using (4.7) in the above equation, and after some elementary calculations, we get

$$
E_{n, q}^{(r)}\left(y_{1}+y_{2}+\cdots+y_{r}\right)=\sum_{\substack{l_{1}, l_{2}, \ldots, l_{r} \geq 0 \\
l_{1}+l_{2}+\cdots+l_{r}=n}}\left(\begin{array}{c}
n \\
l_{1}, l_{2}, \ldots, l_{v}
\end{array}\right) \prod_{j=1}^{r} \int_{\mathbb{Z}_{p}}\left(y_{j}+x_{j}\right)^{l_{j}} d \mu_{-q}\left(x_{j}\right) .
$$

By substituting (2.25) into the above equation, we arrive at the desired result.

By substituting (2.8) into (4.9), then Theorem 4.3 reduces to the following theorem.

Theorem 4.4. For positive integers $n, r$, one has

$$
E_{n, q}^{(r)}\left(y_{1}+y_{2}+\cdots+y_{r}\right)=\sum_{\substack{l_{1}, l_{2}, \ldots, l_{l} \geq 0 \\
l_{1}+l_{2}+\cdots, l_{r}=n}}\left(\begin{array}{c}
n \\
l_{1}, l_{2}, \ldots, l_{r}
\end{array}\right) \prod_{j=1}^{r} \sum_{k=0}^{l_{j}}\left(\begin{array}{l}
l_{j} \\
k
\end{array}\right) y_{j}^{l_{j}-k} E_{l_{j}, q} .
$$

In (4.10), if we replace $y_{1}+y_{2}+\cdots+y_{r}$ by $x$, then we obtain the following corollary.

Corollary 4.5. For $n \geq 0$, one has

$$
\begin{aligned}
E_{n, q}^{(r)}(x) & =\int_{\mathbb{Z}_{p}^{r}}\left(x_{1}+\cdots+x_{r}+x\right)^{m} d \mu_{-q}\left(x_{1}\right) \cdots d \mu_{-q}\left(x_{r}\right) \\
& =\sum_{l_{1}+\cdots+l_{r}+l_{r}+1=m}\left(\begin{array}{c}
m \\
l_{1} \cdots l_{r+1}
\end{array}\right) \int_{\mathbb{Z}_{p}} x_{1}^{l_{1}} d \mu_{-q}\left(x_{1}\right) \cdots \int_{\mathbb{Z}_{p}} x_{r}^{l_{r}} d \mu_{-q}\left(x_{r}\right) x^{l_{r+1}} \\
& =\sum_{l_{1}+\cdots+l_{r}+l_{r}+1=m}\left(\begin{array}{c}
m \\
l_{1} \cdots l_{r+1}
\end{array}\right) E_{l_{1}, q} E_{l_{2}, q} \cdots E_{l_{r}, q} x^{l_{r+1}} .
\end{aligned}
$$

Remark 4.6. By using (4.5)-(4.7), complete sums of products of $q$-Euler polynomials of higher order are also obtained. Proof of Corollary 4.5 was also given by Ryoo et al. [14], which is given by

$$
E_{n, q}^{(r)}(x)=\sum_{k=0}^{n} \sum_{l_{1}+\cdots+l_{r}+l_{r+1}=m}\left(\begin{array}{l}
n \\
k
\end{array}\right)\left(\begin{array}{c}
k \\
l_{1} \cdots l_{r+1}
\end{array}\right) x^{n-k} E_{l_{1}, q} E_{l_{2}, q} \cdots E_{l_{r}, q}
$$

In (4.13), if we take $q \rightarrow 1$, we have

$$
E_{n}^{(r)}(x)=\sum_{l_{1}+\cdots+l_{r}+l_{r+1}=m}\left(\begin{array}{c}
m \\
l_{1} \cdots l_{r+1}
\end{array}\right) E_{l_{1}} E_{l_{2}} \cdots E_{l_{r}} x^{l_{r+1}} .
$$

For more detailed information about complete sums of products of Euler polynomials and Bernoulli polynomials, see also [11, 14, 20-24, 34, 35]. 
Let $\chi$ be a Dirichlet character with conductor $d \in \mathbb{Z}^{+}$. Then

$$
\begin{aligned}
\int_{\mathbb{X}} x(x) e^{t x} d \mu_{-q}(x) & =(q+1) \frac{\sum_{l=0}^{d-1}(-1)^{d-1-l} q^{l} e^{t l} \chi(x)}{e^{d t} q^{d}+1} \\
& =\sum_{n=0}^{\infty} E_{n, X, q} \frac{t^{n}}{n !} .
\end{aligned}
$$
arrive at

By using Taylor expansion of $e^{t x}$ and then comparing coefficients of $t^{n}$ on both sides, we

$$
\int_{\mathbb{X}} x(x) x^{n} d \mu_{-q}(x)=E_{n, x, q}
$$

(cf. [8]).

By (4.16), we have

$$
\begin{aligned}
\int_{\mathbb{X}^{r}} \prod_{i=1}^{r} X\left(x_{i}\right) e^{t\left(x_{1}+\cdots+x_{r}\right)} d \mu_{-q}\left(x_{1}\right) \cdots d \mu_{-q}\left(x_{r}\right) & =\int_{\mathbb{X}^{r}} \prod_{i=1}^{r} x\left(x_{i}\right) e^{t\left(x_{1}+\cdots+x_{r}\right)} d \mu_{-q}\left(x_{1}\right) \cdots d \mu_{-q}\left(x_{r}\right) \\
& =\left(\frac{\sum_{a=0}^{d-1}(-1)^{d-1-a} q^{a} e^{t a} X(a)}{e^{d t} q^{d}+1}\right)^{r} \sum_{j=0}^{r}\left(\begin{array}{l}
r \\
j
\end{array}\right) q^{j} \\
& =\sum_{n=0}^{\infty} E_{n, x, q}^{(r)} \frac{t^{n}}{n !}
\end{aligned}
$$

Thus we give Witt-type formula of $E_{n, x, q}^{(r)}$ as follows.

Theorem 4.7. Let $x$ be a Dirichlet character with conductor $d \in \mathbb{N}$ and let $m \geq 0$. Then

$$
E_{n, x, q}^{(r)}=\int_{\mathbb{X}^{r}}\left(x_{1}+\cdots+x_{r}\right)^{m} \prod_{i=1}^{r} \chi\left(x_{i}\right) d \mu_{-q}\left(x_{1}\right) \cdots d \mu_{-q}\left(x_{r}\right) .
$$

By using (3.2), (2.8), we obtain

$$
E_{n, q, x}^{(r)}=f^{n} \frac{(1+q)^{r}}{\left(1+q^{f}\right)^{r}} \sum_{a_{1}, a_{2}, \ldots, a_{r}=0}^{f-1}(-1)^{a_{1}+\cdots+a_{r}} q^{a_{1}+\cdots+a_{r}} \prod_{k=1}^{r} x\left(a_{k}\right) \sum_{k=0}^{n}\left(\begin{array}{l}
n \\
k
\end{array}\right)\left(\frac{a_{1}+\cdots+a_{r}}{f}\right)^{n-k} E_{k, q^{f}}^{(r)} .
$$

By using (4.7) in the above equation, we have

$$
\begin{aligned}
E_{n, q, x}^{(r)}= & \frac{(1+q)^{r}}{\left(1+q^{f}\right)^{r}} \sum_{a_{1}, a_{2}, \ldots, a_{r}=0}^{f-1}(-1)^{a_{1}+\cdots+a_{r}} q^{a_{1}+\cdots+a_{r}} \prod_{k=1}^{r} x\left(a_{k}\right) \\
& \times \sum_{k=0}^{n} \sum_{\substack{l_{1}, l_{2}, \ldots, l_{v} \geq 0 \\
l_{1}+l_{2}+\cdots+l_{v}=n-k}}\left(\begin{array}{c}
n-k \\
l_{1}, l_{2}, \ldots, l_{v}
\end{array}\right)\left(\begin{array}{l}
n \\
k
\end{array}\right) \prod_{y=1}^{v} a_{y}^{l_{y}} f^{k} E_{k, q^{f}}^{(r)} .
\end{aligned}
$$




\section{Acknowledgments}

The first and the second authors are supported by the research fund of Uludag University Projects no. F-2006/40 and F-2008/31. The third author is supported by the research fund of Akdeniz University. The authors would like to thank the referee for their comments.

\section{References}

[1] T. Kim, "On the q-extension of Euler and Genocchi numbers," Journal of Mathematical Analysis and Applications, vol. 326, no. 2, pp. 1458-1465, 2007.

[2] T. Kim, "On $p$-adic interpolating function for $q$-Euler numbers and its derivatives," Journal of Mathematical Analysis and Applications, vol. 339, no. 1, pp. 598-608, 2008.

[3] T. Kim, "A note on some formulae for the q-Euler numbers and polynomials," Proceedings of the Jangjeon Mathematical Society, vol. 9, no. 2, pp. 227-232, 2006.

[4] T. Kim, "A note on $p$-adic invariant integral in the rings of $p$-adic integers," Advanced Studies in Contemporary Mathematics, vol. 13, no. 1, pp. 95-99, 2006.

[5] T. Kim, "q-generalized Euler numbers and polynomials," Russian Journal of Mathematical Physics, vol. 13, no. 3, pp. 293-298, 2006.

[6] T. Kim and J.-S. Cho, "A note on multiple Dirichlet's q-l-function," Advanced Studies in Contemporary Mathematics, vol. 11, no. 1, pp. 57-60, 2005.

[7] T. Kim, "A note on the q-multiple zeta function," Advanced Studies in Contemporary Mathematics, vol. 8, no. 2, pp. 111-113, 2004.

[8] T. Kim, M.-S. Kim, L. Jang, and S.-H. Rim, “New $q$-Euler numbers and polynomials associated with p-adic q-integrals," Advanced Studies in Contemporary Mathematics, vol. 15, no. 2, pp. 243-252, 2007.

[9] T. Kim, "On Euler-Barnes multiple zeta functions," Russian Journal of Mathematical Physics, vol. 10, no. 3, pp. 261-267, 2003.

[10] T. Kim, “q-Volkenborn integration,” Russian Journal of Mathematical Physics, vol. 9, no. 3, pp. 288-299, 2002.

[11] T. Kim, "Sums of products of q-Bernoulli numbers," Archiv der Mathematik, vol. 76, no. 3, pp. 190-195, 2001.

[12] T. Kim, S.-H. Rim, and Y. Simsek, "A note on the alternating sums of powers of consecutive q-integers," Advanced Studies in Contemporary Mathematics, vol. 13, no. 2, pp. 159-164, 2006.

[13] T. Kim and S.-H. Rim, "New Changhee $q$-Euler numbers and polynomials associated with $p$-adic q-integrals," Computers \& Mathematics with Applications, vol. 54, no. 4, pp. 484-489, 2007.

[14] C. S. Ryoo, L. Jang, and T. Kim, "Note on q-extensions of Euler numbers and polynomials of higher order," to appear in Journal of Inequalities and Applications.

[15] H. Ozden, Y. Simsek, S.-H. Rim, and I. N. Cangul, "A note on p-adic $q$-Euler measure," Advanced Studies in Contemporary Mathematics, vol. 14, no. 2, pp. 233-239, 2007.

[16] H. Ozden and Y. Simsek, "A new extension of $q$-Euler numbers and polynomials related to their interpolation functions," to appear in Applied Mathematics Letters.

[17] H. Ozden, Y. Simsek, and I. N. Cangul, "Euler polynomials associated with $p$-adic $q$-Euler measure," General Mathematics, vol. 15, no. 2-3, pp. 24-37, 2007.

[18] H. Ozden, I. N. Cangul, and Y. Simsek, "Generating functions of the $(h, q)$-extension of Euler polynomials and numbers," to appear in Acta Mathematica Hungarica.

[19] H. Ozden and Y. Simsek, "Interpolation function of the $(h, q)$-extension of twisted Euler numbers," to appear in Computers \& Mathematics with Applications.

[20] H. Ozden, Y. Simsek, and I. N. Cangul, "Remarks on sum of products of $(h, q)$-twisted Euler polynomials and numbers," to appear in Journal of Inequalities and Applications.

[21] S.-H. Rim and T. Kim, "Explicit $p$-adic expansion for alternating sums of powers," Advanced Studies in Contemporary Mathematics, vol. 14, no. 2, pp. 241-250, 2007.

[22] J. Satoh, "Sums of products of two q-Bernoulli numbers," Journal of Number Theory, vol. 74, no. 2, pp. 173-180, 1999.

[23] Y. Simsek, "Complete sum of products of $(h, q)$-extension of Euler polynomials and numbers," submitted.. 
[24] Y. Simsek, V. Kurt, and D. Kim, "New approach to the complete sum of products of the twisted $(h, q)$-Bernoulli numbers and polynomials," Journal of Nonlinear Mathematical Physics, vol. 14, no. 1, pp. 44-56, 2007.

[25] Y. Simsek, "Twisted $(h, q)$-Bernoulli numbers and polynomials related to twisted $(h, q)$-zeta function and L-function," Journal of Mathematical Analysis and Applications, vol. 324, no. 2, pp. 790-804, 2006.

[26] Y. Simsek, "On $p$-adic twisted $q$-L-functions related to generalized twisted Bernoulli numbers," Russian Journal of Mathematical Physics, vol. 13, no. 3, pp. 340-348, 2006.

[27] Y. Simsek, " $q$-analogue of twisted $l$-series and $q$-twisted Euler numbers," Journal of Number Theory, vol. 110, no. 2, pp. 267-278, 2005.

[28] H. M. Srivastava, T. Kim, and Y. Simsek, " $q$-Bernoulli numbers and polynomials associated with multiple q-zeta functions and basic L-series," Russian Journal of Mathematical Physics, vol. 12, no. 2, pp. 241-268, 2005.

[29] T. Kim, " $q$-Euler numbers and polynomials associated with $p$-adic $q$-integrals," Journal of Nonlinear Mathematical Physics, vol. 14, no. 1, pp. 15-27, 2007.

[30] I. N. Cangul, V. Kurt, Y. Simsek, H. K. Pak, and S.-H. Rim, "An invariant $p$-adic $q$-integral associated with $q$-Euler numbers and polynomials," Journal of Nonlinear Mathematical Physics, vol. 14, no. 1, pp. 8-14, 2007.

[31] T. Kim, "A note on $p$-adic $q$-integrals associated with $q$-Euler numbers," Advanced Studies in Contemporary Mathematics, vol. 15, pp. 133-137, 2007.

[32] L. Comtet, Advanced Combinatorics, D. Reidel, Dordrecht, The Netherlands, 1974.

[33] R. L. Graham, D. E. Knuth, and O. Patashnik, Concrete Mathematics, Addison-Wesley, Reading, Mass, USA, 1989.

[34] K. Dilcher, "Sums of products of Bernoulli numbers," Journal of Number Theory, vol. 60, no. 1, pp. 23-41, 1996.

[35] Z.-W. Sun and H. Pan, "Identities concerning Bernoulli and Euler polynomials," Acta Arithmetica, vol. 125, no. 1, pp. 21-39, 2006. 


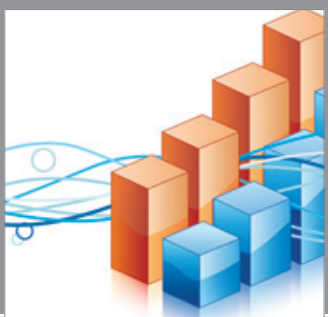

Advances in

Operations Research

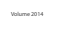

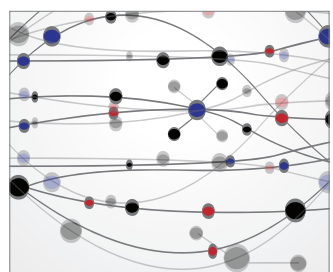

\section{The Scientific} World Journal
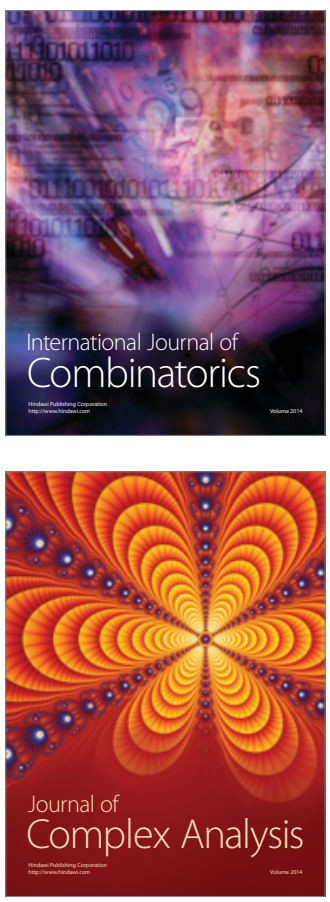

International Journal of

Mathematics and

Mathematical

Sciences
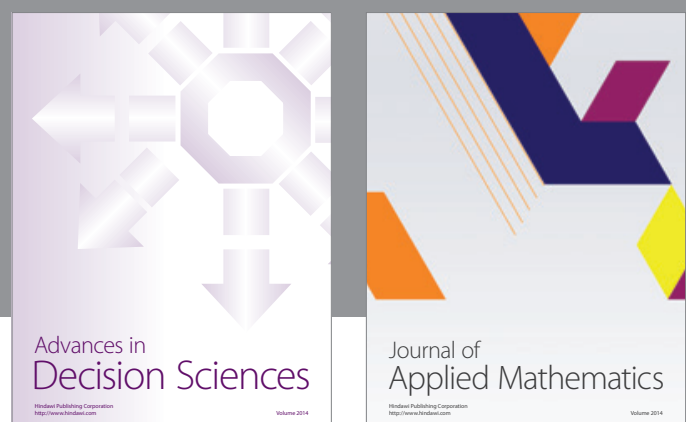

Journal of

Applied Mathematics
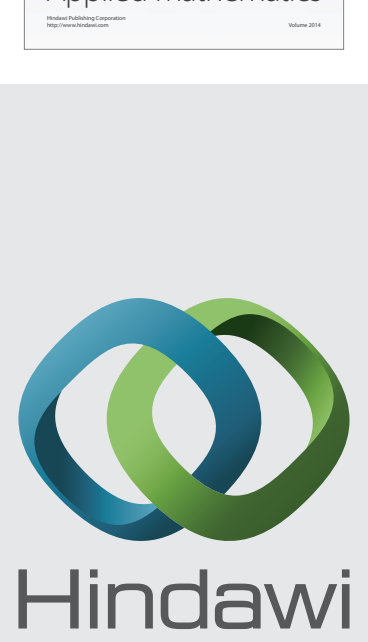

Submit your manuscripts at http://www.hindawi.com
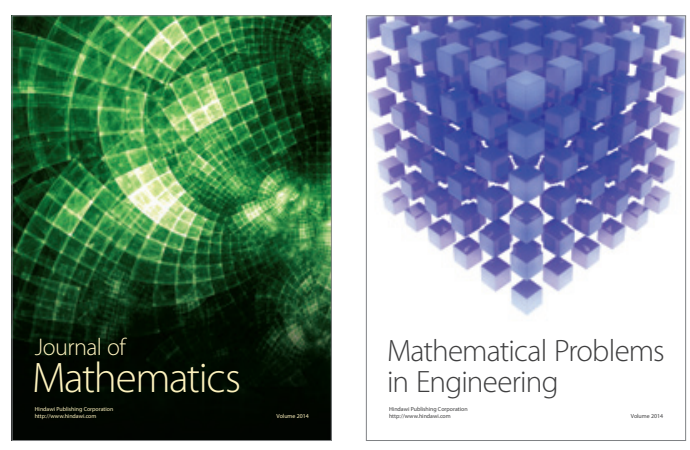

Mathematical Problems in Engineering
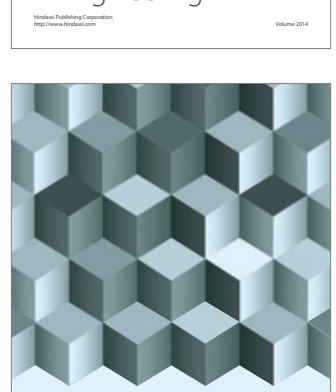

Journal of

Function Spaces
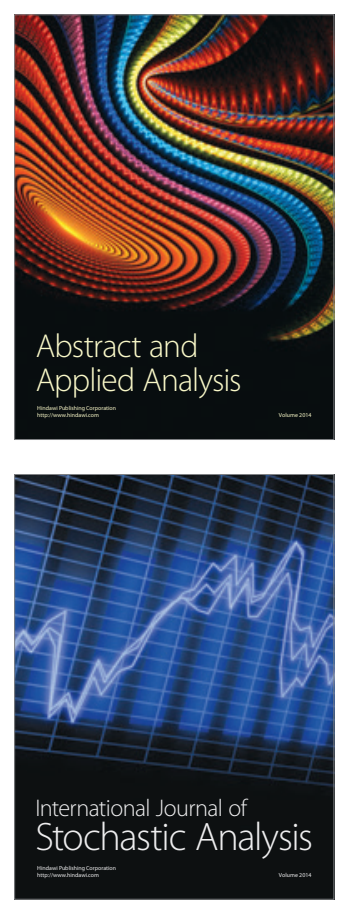

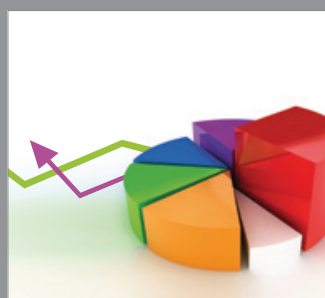

ournal of

Probability and Statistics

Promensencen
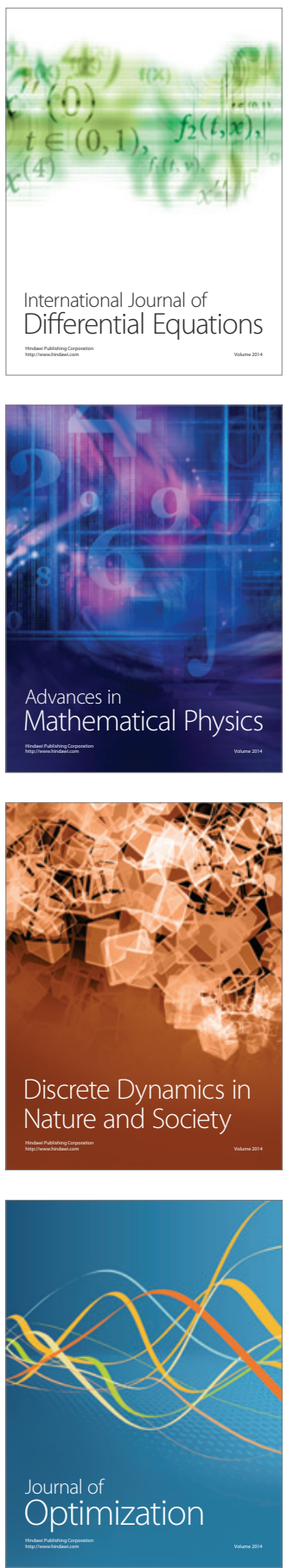